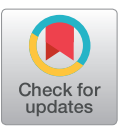

\section{Case Report}

\title{
Microsurgical treatment of lateral cavernous sinus wall dural arteriovenous fistula with large venous aneurysm: A case report
}

\author{
Dong Jin Kim¹, Wonhyoung Park', Jung Cheol Park', Jae Sung Ahn', \\ Deok Hee Lee ${ }^{2}$, Joonho Byun ${ }^{1}$ \\ ${ }^{7}$ Department of Neurosurgery, Asan Medical Center, University of Ulsan, College of Medicine, Seoul, Korea \\ ${ }^{2}$ Department of Radiology, Asan Medical Center, University of Ulsan, College of Medicine, Seoul, Korea
}

\section{$\mathrm{J}$ Cerebrovasc Endovasc Neurosurg. 2021 March;23(1):41-48}

Received: 5 August 2020

Revised: 25 August 2020

Accepted: 11 September 2020

\section{Correspondence to} Joonho Byun

Department of Neurosurgery, Asan Medical Center, University of Ulsan, College of Medicine, 88 Olympic-ro 43-gil, Songpa-gu, Seoul 05505, Korea

Tel +82-2-3010-3550

Fax +82-2-476-6738

E-mail drjunho@naver.com ORCID http://orcid.org/0000-0003-0687-3286
We report a case of lateral cavernous sinus wall dural arteriovenous fistula (DAVF) accompanied large venous aneurysm which is presented intracerebral hemorrhage (ICH). A 58-year-old male patient came to emergency department for acute onset of headache and dysarthria. In brain computed tomography scan, large left temporal lobe ICH was noted. In transfemoral cerebral angiography, multiple arteries from external carotid artery and left internal carotid artery (ICA) fed arteriovenous shunt. This shunt was drained through cavernous sinus with enlarged multiple cortical veins. One large venous aneurysm was estimated as bleeding focus for $\mathrm{ICH}$. Considering $\mathrm{ICH}$ and high flow shunt, we planned urgent treatment to reduce flow of arteriovenous shunt. However, transvenous embolization was failed due to tortuous venous anatomy. Therefore, we planned craniotomy and microsurgical treatment. There was engorged small vessel in lateral wall of cavernous sinus and vascular trunk which is fistulous connection was noted. Fistula connection was obliterated and disconnected after coagulation. In postoperative image, fistula was completely disappeared and there was no cortical venous reflux, also large venous aneurysm was disappeared Patient recovered very well without new neurological deficits. We reported successfully treated lateral cavernous sinus wall DAVF by combined endovascular and transcranial-microsurgical treatment.

Keywords Dural arteriovenous fistula, Microsurgery, Cavernous sinus, Venous aneurysm
This is an Open Access article distributed under the terms of the Creative Commons Attribution Non-Commercial License (http://creativecommons.org/licenses/ by-nc/3.0/) which permits unrestricted noncommercial use, distribution, and reproduction in any medium, provided the original work is properly cited.

\section{INTRODUCTION}

Dural arteriovenous fistula (DAVF) is pathologic connection between dural arteries and venous sinuses, meningeal veins or cortical veins. DAVF account for $10-15 \%$ of intracranial vascular malformation. ${ }^{4)}$ DAVFs are most commonly located in the tentorium, 
JCEN

transverse-sigmoid sinuses and inside of cavernous sinus. ${ }^{3)}$ DAVF elevates venous pressure, it leads retrograde flow of venous sinuses and venous reflux to cortical veins (cortical venous drainage, CVD). Clinical presentation of DAVF is vary, it depends on hemodynamic properties and location. Hemorrhagic presentation is more common in high grade DAVF (Borden type III), and high grade DAVF needs prompt endovascular or surgical intervention. ${ }^{1)}$ We report a case of lateral cavernous sinus wall DAVF accompanied large venous aneurysm in superficial middle cerebral vein which is presented intracerebral hemorrhage (ICH) and successfully treated with combined endovascular and microsurgery.
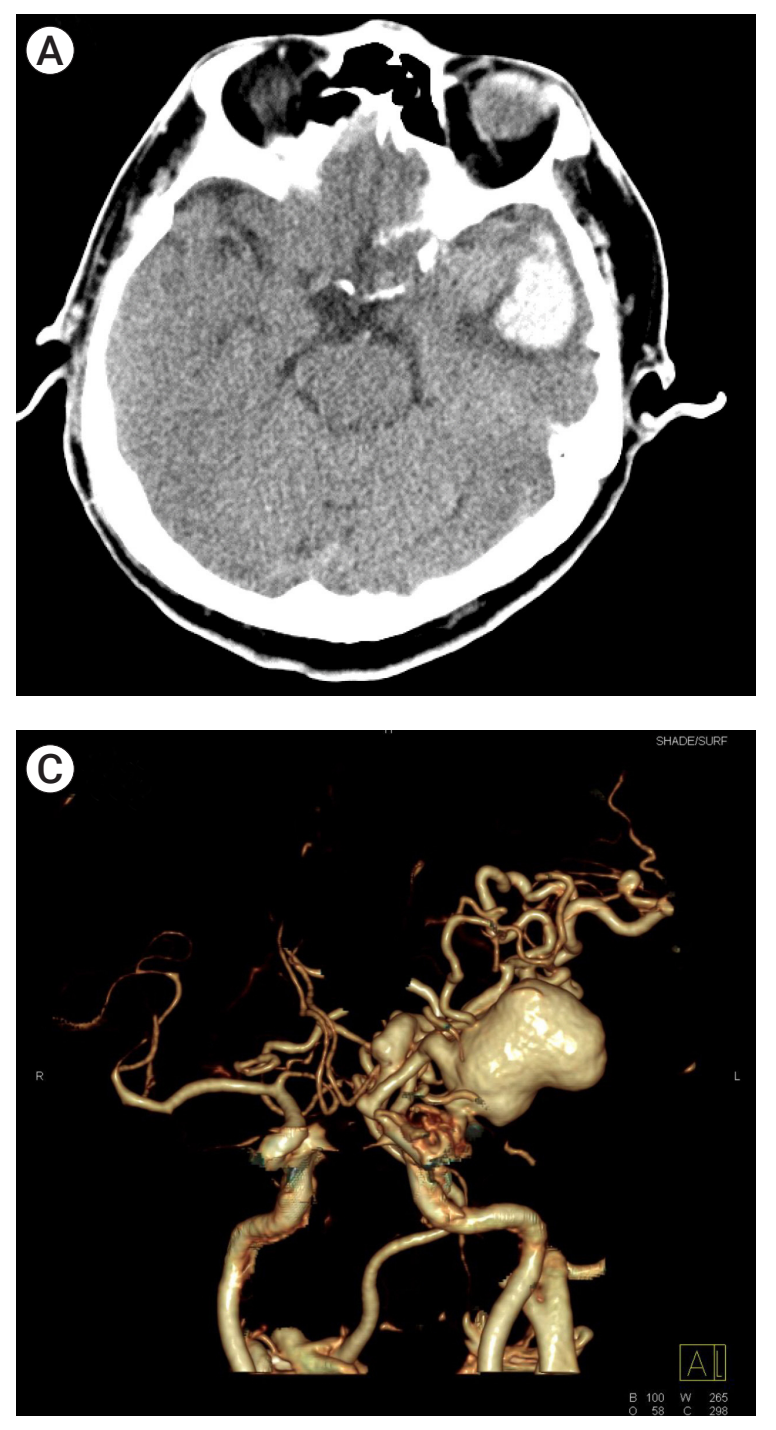

\section{CASE DESCRIPTION}

\section{Initial history and examination}

This 58-year-old male patient came to emergency department for acute onset of headache and dysarthria. He didn't have any past medial history including trauma. In brain computed tomography (CT) scan, large left temporal lobe ICH and high-density lesion was noted and enlarged abnormal vascular structure in contrast enhanced image. In CT angiography, large ectatic venous abnormalities and venous aneurysm were seen around lateral wall of the left cavernous sinus (Fig. 1A, B, C).

Urgent transfemoral cerebral angiography was performed with clinical impression of DAVF or arteriove-

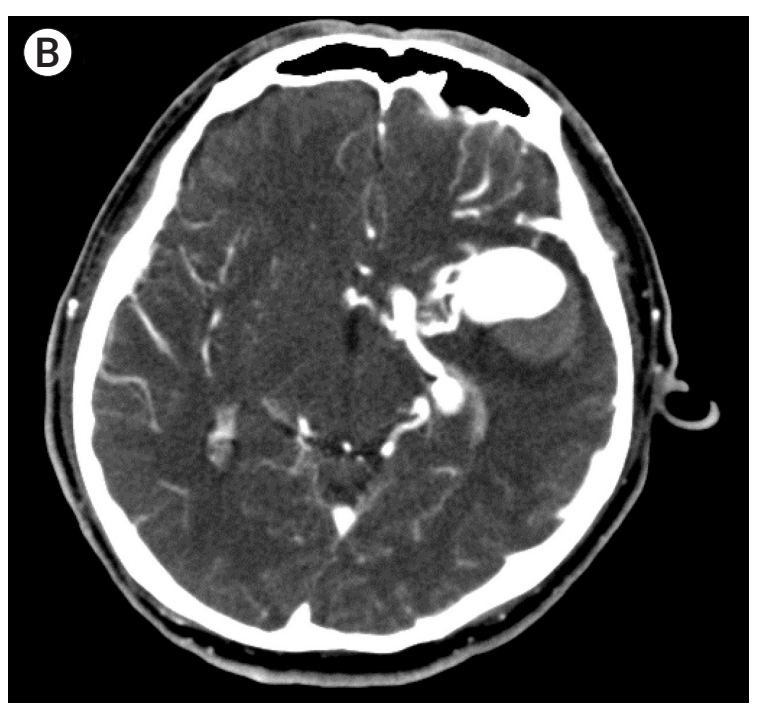

Fig. 1. Initial brain computed tomography (CT) angiography of lateral cavernous sinus wall dural arteriovenous fistula. (A) Left temporal lobe intracerebral hemorrhage was seen on non-contrast brain CT scan. Slight high density lesion was located in medial portion of hematoma. (B), (C) Contrast enhanced CT scan showed engorged venous structure and large venous aneurysm in anterior and medial temporal lobe. Complex interlacing vascular structure were seen in lateral area of cavernous segment of internal carotid artery. 
nous malformation with high flow shunt. Middle meningeal and accessory meningeal arteries from external carotid artery (ECA) fed arteriovenous shunt, shunt was also fed by inferolateral trunk of left internal carotid artery (ICA) and recurrent branches from ophthalmic artery. This shunt was drained through common venous trunk in superolateral aspect of cavernous sinus wall with enlarged multiple cortical veins including super- ficial middle cerebral vein and basal vein of Rosenthal. One large venous aneurysm in the superficial middle cerebral vein was estimated as bleeding focus for right temporal lobe ICH (Fig. 2A, B, C, D).

Patient was finally diagnosed DAVF in lateral wall of cavernous sinus. Considering ICH (ruptured DAVF) and high flow shunt, we planned urgent glue embolization of middle meningeal and accessory meningeal
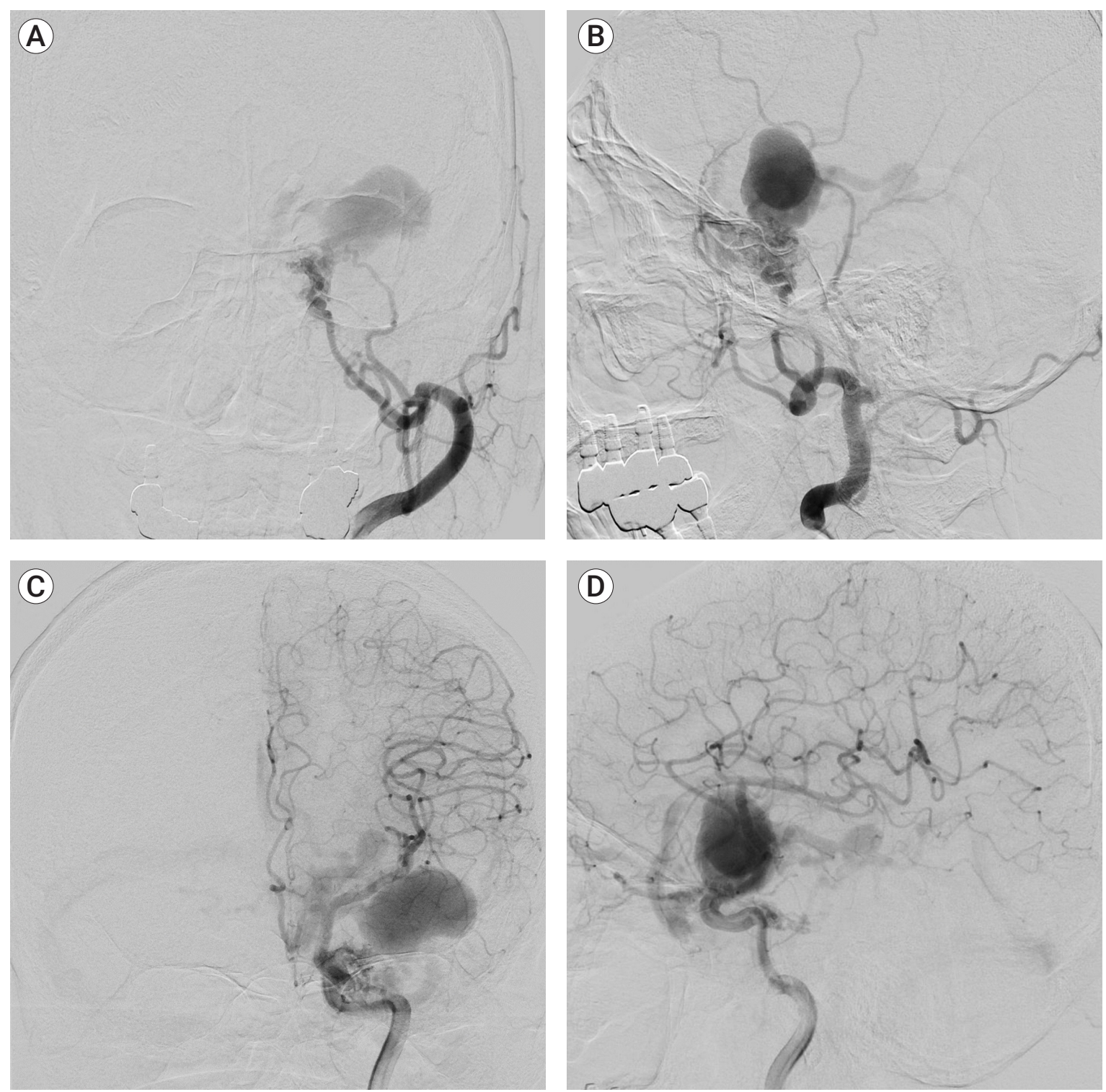

Fig. 2. Initial cerebral angiographic findings of lateral cavernous sinus wall dural arteriovenous fistula (DAVF). (A), (B) External carotid artery angiogram: DAVF was fed by middle meningeal and accessory arteries. Shunt flow was drained by basal vein of Rosenthal and superficial middle cerebral vein. (C), (D) Internal carotid artery angiogram: inferolateral trunk of cavernous segment of internal carotid artery and recurrent branches of ophthalmic artery. Middle cerebral artery was displaced superiorly. Large venous aneurysm of superficial middle cerebral vein was seen. 
arteries to reduce flow of arteriovenous shunt (Fig. 3A, B, C, D, E and F).

\section{Treatment of DAVF}

We discussed radical treatment of this patient's DAVF with neuroradiologist and neurointerventionist. Firstly, we attempted transvenous embolization through trans- verse sinus and vein of Labbe. However, embolization was failed due to tortuous venous anatomy and acute angulation between transverse and tentorial sinus (Fig. 3E). However, for decreasing amount of arteriovenous shunt flow, coil embolization of feeding arteries from inferolateral trunk was performed to reduce blood loss that may occur during microsurgery (Fig. 3F).
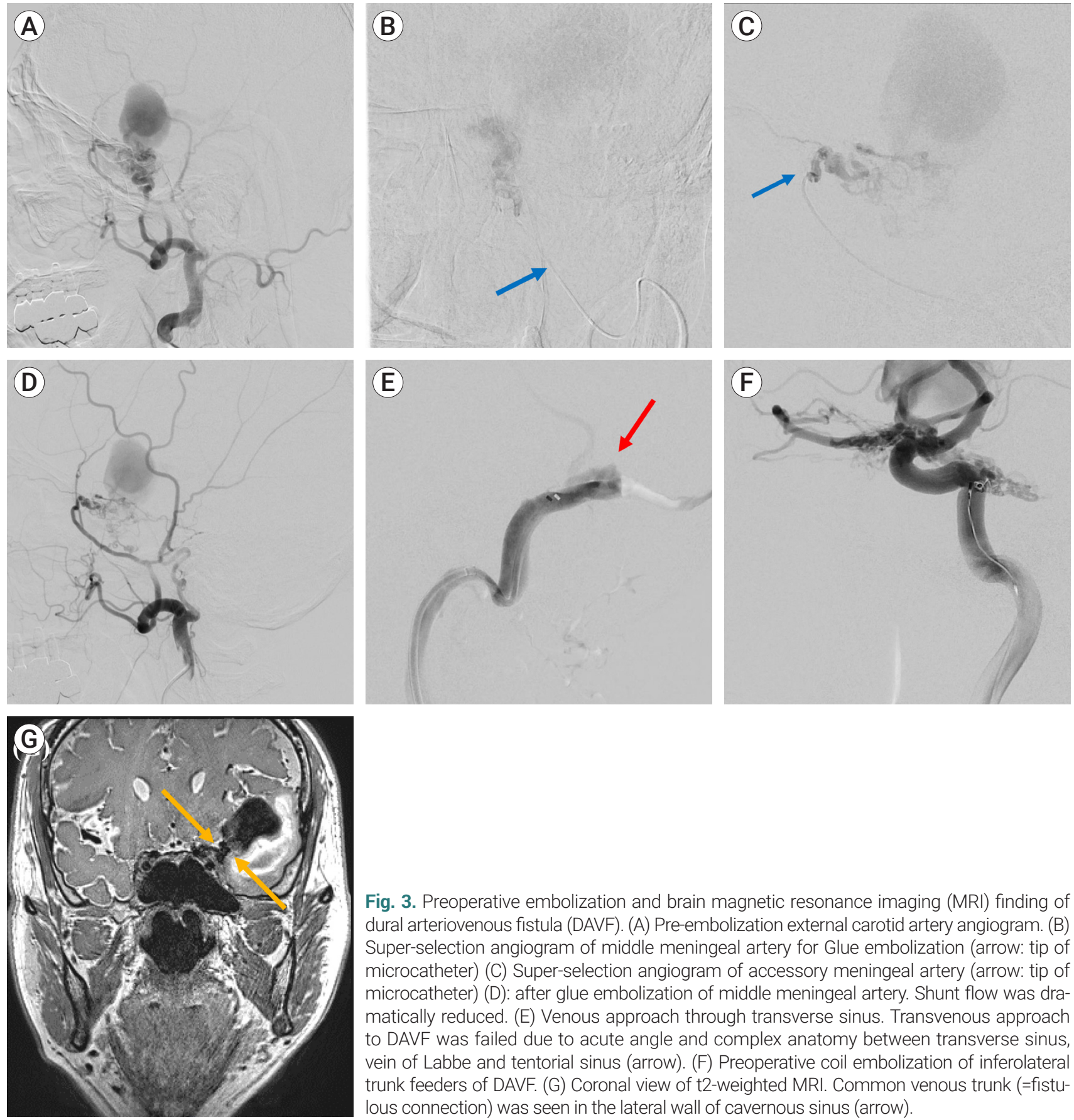

Fig. 3. Preoperative embolization and brain magnetic resonance imaging (MRI) finding of dural arteriovenous fistula (DAVF). (A) Pre-embolization external carotid artery angiogram. (B) Super-selection angiogram of middle meningeal artery for Glue embolization (arrow: tip of microcatheter) (C) Super-selection angiogram of accessory meningeal artery (arrow: tip of microcatheter) (D): after glue embolization of middle meningeal artery. Shunt flow was dramatically reduced. (E) Venous approach through transverse sinus. Transvenous approach to DAVF was failed due to acute angle and complex anatomy between transverse sinus, vein of Labbe and tentorial sinus (arrow). (F) Preoperative coil embolization of inferolateral trunk feeders of DAVF. (G) Coronal view of t2-weighted MRI. Common venous trunk (=fistulous connection) was seen in the lateral wall of cavernous sinus (arrow). 
Microsurgical disconnection of DAVF was planned. Frontotemporal craniotomy with zygomatic osteotomy for wide exposure from frontal to temporal skull base was performed. Carotid, chiasmatic, medial sylvian cistern arachnoid dissection was performed. Optic nerve, ICA and enlarged vein in optico-carotid triangle were exposed. There was engorged small vessel in lateral wall of cavernous sinus and fistulous connected vascular trunk was noted. Arteriovenous shunt flow through venous trunk was confirmed using indocyanine green (ICG) videoangiography (Fig. 4F). Fistula connection was obliterated using permanent aneurysm clip and disconnected after coagulation. Small temporal corticectomy was performed to evacuate $\mathrm{ICH}$, and venous aneurysm was shrunken after disconnection of fistula (Fig. 4E). Further exploration from cavernous
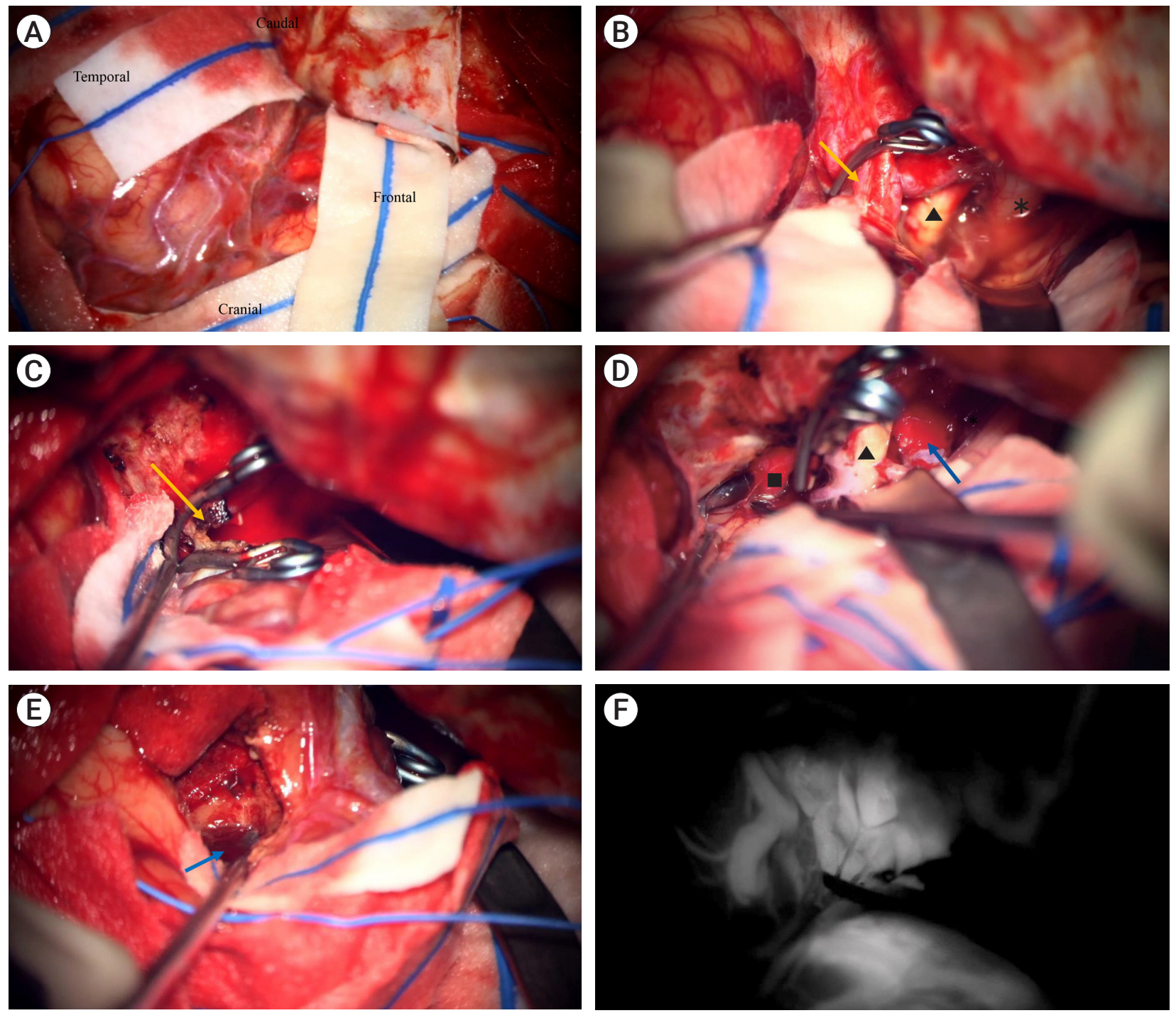

Fig. 4. Intraoperative photography of lateral cavernous sinus wall dural arteriovenous fistula. (A) Frontotemporal zygomatic osteotomy was performed for skull base approach. (B) Fistulous connection was seen in lateral area of internal carotid artery. It located in the lateral wall of cavernous sinus. Engorged vessel was seen in the surface of lateral wall of cavernous sinus. Vascular clip was placed in the connection of fistula. (C) Fistula connection was disconnected after coagulation. (D) Further exploration was performed temporal base dura and tentorial edge. There was no additional abnormal vascular structure. (arrow: one enlarged branch of basal vein). (E) Evacuation of intracerebral hematoma of temporal lobe. (arrow: Shrunken wall of venous aneurysm was noted in hematoma cavity). (F) Indocyanine green videoangiogram of venous trunk. Venous trunk and reflux venous flow through superficial middle cerebral vein were visualized in early arterial phase. *: optic nerve, $\mathbf{\Delta}$ : internal carotid artery, $\mathbf{\square}$ : oculomotor nerve. 
sinus superior wall to tentorial edge and tentorium was done, there was no additional fistulous connection (Fig. 4A, B, C and D).

Postoperative CT scan and angiography were performed to confirm complete disconnection of DAVF. Fistula was completely disappeared in postoperative CT scan and angiography. Also, there was no cortical venous reflux and large venous aneurysm was disappeared (Fig. 5A, B, C, D, E, and F). Patient was recovered soon after surgery and discharged to home on postoperative 7 days without neurological deficits.

\section{DISCUSSION}

There were some reports of DAVF in around anterior clinoid process. ${ }^{8) 9)}$ Also, there was report of sphenoid wing DAVF. ${ }^{10)}$ However, in our case, common venous trunk located in the middle of the lateral wall of cavernous sinus, fed by both ECA and ICA. Also, large venous aneurysm was accompanied due to increased venous pressure. Cavernous sinus DAVF usually complaint symptoms of headache and eyeball symptoms including exophthalmos, chemosis, also reflux blood go through the superior ophthalmic vein (SOV). However, in our case, fistulous connection was located in the lateral wall of cavernous sinus, not inside of cavernous sinus and patient did not show any symptoms including ocular symptom before bleeding from DAVF. Also, venous blood was reflux through not SOV but superficial middle cerebral vein and basal vein of Rosenthal.

In this case, main feeders of DAVF were middle meningeal artery, accessory meningeal artery, artery of foramen rotundum from ECA. And inferolateral trunk,
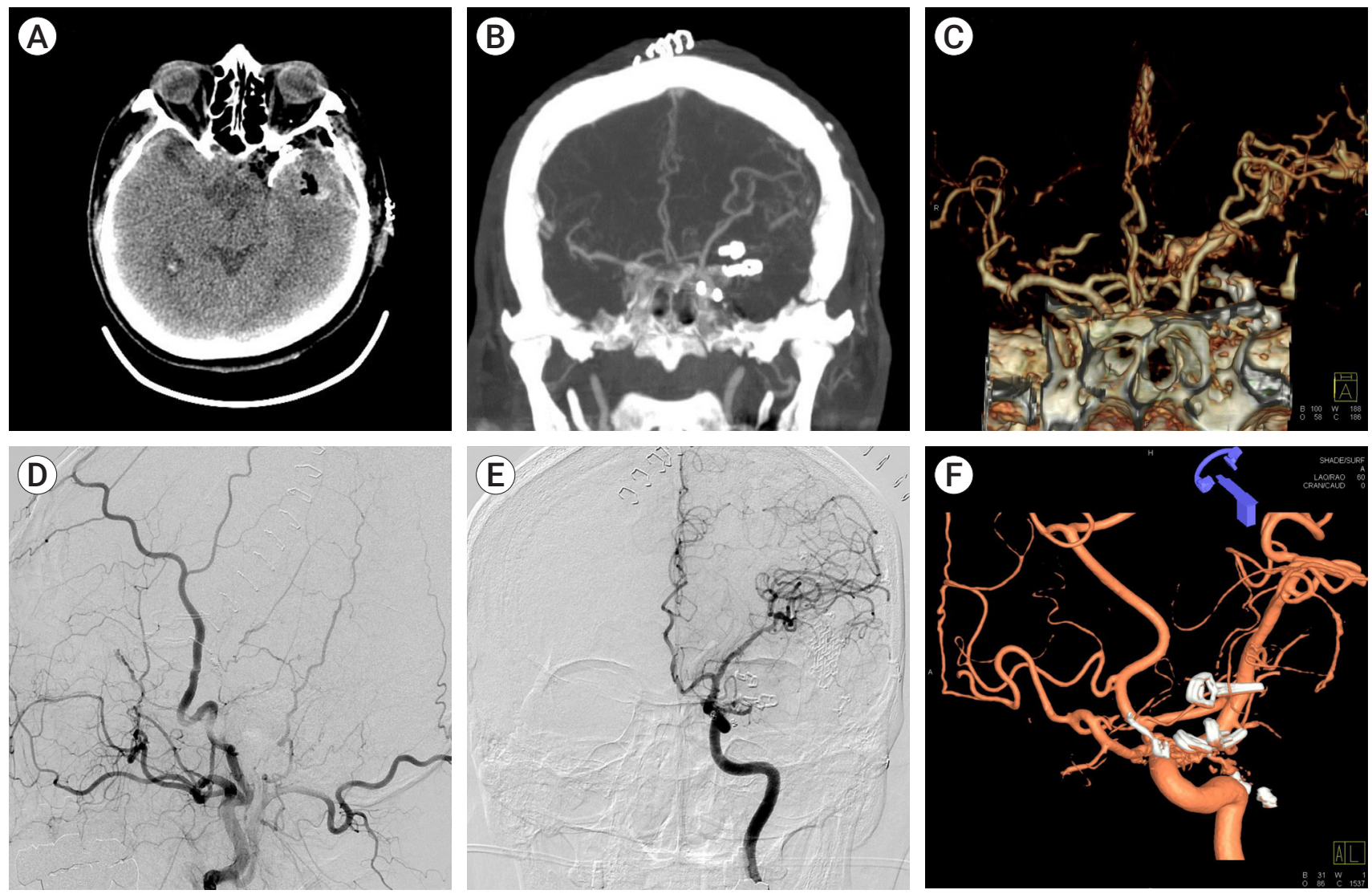

Fig. 5. Postoperative brain computed tomography (CT) and trans femoral cerebral angiography (TFCA). (A-C) Postoperative CT scan: Temporal lobe hematoma was totally removed. Engorged vein and venous aneurysm could not be seen on the postoperative images. (D-F) Postoperative TFCA: complete disconnection of dural arteriovenous fistula was confirmed on both external and internal carotid artery angiograms. 
recurrent ethmoidal branches of ophthalmic artery from ICA also fed DAVF. DAVF was drained to superficial middle cerebral vein and basal vein of Rosenthal. There was not only multiple cortical venous reflux but also, large venous aneurysm which was cause of intracerebral hemorrhage. This DAVF classified as Borden type III which should be treated to prevent hemorrhage.

Treatment options of DAVF are endovascular treatment, microsurgery and radiosurgery. Microsurgical disconnection of the DAVF is not usually considered first-line treatment in the endovascular era. However, disconnection of fistula through microsurgery may be considered the most curative treatment for DAVF. Coagulation of arterial feeders and excision of dura or disconnection of fistulous connection are usual microsurgical strategy. However, surgical morbidity, especially significant blood loss should be considered in microsurgical treatment. ${ }^{1)}$ Intraoperative indocyanine green videoangiography and microvascular doppler ultrasound are essential adjunctive tool in DAVF microsurgery.

Endovascular treatment becomes the mainstay role in therapy of DAVF in the endovascular era. Transarterial embolization (TAE) is more commonly considered. ${ }^{1)}$ TAE of DAVF has been shown good treatment outcomes. ${ }^{25)}$ Feasibility of super-selection of multiple feeding arteries is most important consideration in TAE. Also, caution should be taken accidental embolization of the draining venous system, it may result venous infarction and intracranial hemorrhage. ${ }^{2) 77}$ Moreover, proximal occlusion of feeding arteries allows the recruitment of feeding arteries of DAVF and residual arteriovenous shunt. Transvenous embolization (TVE) is used for DAVF which fed by multiple small arterial feeders or very tortuous course of feeding arteries. ${ }^{6}$ Occlusion of proximal segment of draining vein can lead the obliteration of DAVF. However, TVE also harbors risks of venous occlusion, venous perforation, venous infarction and intracranial hemorrhage. $\left.{ }^{4}\right)$ The course of drainage system could affect the approach to target area, long serpentine veins or acute angulation of venous tributaries make transvenous approach complicated. In addition, occlusion of distal segment of venous system without proximal segment could lead recruitment of feeders which can cause recanalization of fistula. Although, there are some limitations, endovascular treatment of DAVF has strong advantages of less invasiveness and approach to inoperable conditions. Also, endovascular treatment can be a good adjunctive tool for microsurgery.

In this case, emergent glue embolization of feeding arteries from ECA was performed to reduce pressure of ruptured large venous aneurysm. Thereafter, curative TVE was attempted, however, approach to vein of Labbe was failed due to complexity of venous anatomy of transverse-tentorial sinus and acute angulation of junction of vein of Labbe. The last option of curative treatment was microsurgical disconnection in this case. To wide exposure and exploration, frontotemporal and zygomatic approach was performed. After sylvian arachnoid dissection, we easily found fistulous connection in lateral wall of cavernous sinus and it was disconnected using permanent aneurysm clip. Additional coagulation of engorged small vessels in the lateral wall of cavernous sinus. Total blood loss during surgery was less than $200 \mathrm{~mL}$, preoperative proximal occlusion of feeders from both ECA and ICA via endovascular approach would be helpful for intraoperative blood loss.

In high grade and complex DAVF, in some cases, multidisciplinary team approach for treatment should be needed. Preoperative embolization can reduce amount of shunt flow and urgently treat high risk lesion of DAVF. In our case, although endovascular treatment could not be curative, it may give the time to make precise microsurgical plan and minimize blood loss during microsurgery. This Combined endovascular and microsurgical treatment lead excellent treatment outcome of complex DAVF.

\section{CONCLUSIONS}

Lateral cavernous sinus wall DAVF is relatively rare. We reported successfully treated lateral cavernous sinus wall DAVF by combined endovascular and transcranial-microsurgical treatment. 


\section{Acknowledgements}

We are indebted to neuroradiologists in our institute, who are always cooperative with us to treat complex neurosurgical disease.

\section{Disclosure}

The authors report no conflict of interest concerning the materials or methods used in this study or the findings specified in this paper.

\section{REFERENCES}

1. Borden JA, Wu JK, Shucart WA. A proposed classification for spinal and cranial dural arteriovenous fistulous malformations and implications for treatment. J Neurosurg. 1995 Feb;82(2):166-79.

2. Cognard C, Januel AC, Silva NA Jr, Tall P. Endovascular treatment of intracranial dural arteriovenous fistulas with cortical venous drainage: new management using Onyx. AJNR Am J Neuroradiol. 2008 Feb;29(2):235-41.

3. Daniels DJ, Vellimana AK, Zipfel GJ, Lanzino G. Intracranial hemorrhage from dural arteriovenous fistulas: clinical features and outcome. Neurosurg Focus. 2013 May;34(5):E15.

4. Gandhi D, Chen J, Pearl M, Huang J, Gemmete JJ, Kathuria
S. Intracranial dural arteriovenous fistulas: classification, imaging findings, and treatment. AJNR Am J Neuroradiol. 2012 Jun;33(6):1007-13.

5. Lamin S, Chew HS, Chavda S, Thomas A, Piano M, Quilici L, et al. Embolization of intracranial dural arteriovenous fistulas using PHIL liquid embolic agent in 26 patients: a multicenter study. AJNR Am J Neuroradiol. 2017 Jan;38(1):127-31.

6. Roy D, Raymond J. The role of transvenous embolization in the treatment of intracranial dural arteriovenous fistulas. Neurosurgery. 1997 Jun;40(6):1133-41;discussion 1141-4.

7. Tomak PR, Cloft HJ, Kaga A, Cawley CM, Dion J, Barrow DL. Evolution of the management of tentorial dural arteriovenous malformations. Neurosurgery. 2003 Apr;52(4):750-60;discussion 60-2.

8. Uchiyama T, Horiuchi T, Murata T, Hongo K. Dural arteriovenous fistula between inferolateral trunk of the internal carotid artery and superficial sylvian vein. Neurol Med Chir (Tokyo). 2011 Sep;51(9):642-4.

9. Ushikoshi S, Honma T, Uchida K, Yasuda H, Ajiki M. Dural arteriovenous fistula at the anterior clinoid process draining directly into the superficial middle cerebral vein. Neurol Med Chir (Tokyo). 2013 Mar;53(3):195-8.

10. Watanabe J, Maruya J, Nishimaki K. Surgical treatment of a dural arteriovenous fistula in the sphenoid wing with a unique drainage pattern through the basal vein of Rosenthal. NMC Case Rep J. 2015 Jun;2(3):88-92. 